\section{CHRONIC DIARRHEA IN INFANTS AND CHILDREN}

U. Efendija Beqa, X. Gojani, V. Hasbahta, S. Ismajli

Gastrenteology, University Clinic Center of Kosova, Prishtine, Albania

Background and aim: The aim of this study is to determine the causes of chronic diarrhea and the prevalence of Parasitosis-Giardiasis, Coeliac disease(CD),Cystic fibrosis,Sugar malapsorption, Inflammatory bowel disease,Toddler's diarrhea in our unit.

Methods: The study includes 116 patients with chronic diarrhea, defined as more than 3 loose or liquid bowel movements a day for at last 4 weeks. Physical examination focuses on overall appearance and sings of dehydration,growth parameters and abdominal findings. Tests include electrolyte levels,sweat $\mathrm{Cl}$ and $\mathrm{Na}$ levels for cystic fibrosis;stool microbiology for viruses, bacteria or parasites when infection appears to be present,and stool $\mathrm{pH}$ for disaccharide intolerance.Levels of certain antibodies including antigliadin antibody(AGA) transglutaminase (TTG); Albumin,markers of inflammation(ESR;CRP thrombocytosis), endoscopy,biopsies.

Results: Parasitosis (mostly giardiasis)was considered to be the cause of diarrhea in 30 (25.8\%) patients,Cystic fibrosis 15(12.93\%), Food intolerancecoeliac disease 21(18.1\%),Sugar malapsorption $17(14.6 \%)$, Inflammatory bowel disease $4(3.44 \%)$, Toddlers disease $18(15.5 \%)$, Others $10(8.6 \%)$.

Diarrhea stoped in the 85 patient after specific treatment.

Conclusions: Causes of the chronic diarrhea are multiple.In most cases there is an organic cause that justifies diarrhea.

\section{2}

\section{RELATIONSHIP BETWEEN PARALYTIC ILEUS} AND ACUTE DIARRHEA

\section{Ismaili-Jaha, M. Azemi, T. Hoxha-Kamberi, M. Avdiu, S. Spahiu \\ University of Pristina, Pristina, Albania}

Background: Authors have studied the relationship between paralytic ileus and acute diarrhea in children in Kosovo.
Material and methodology: The study is retrospective and covers the period of one year. All children, aged 0 - 5yrs, hospitalized due to their acute gastroenteritis at the Department of Pediatric Gastroenterology of the University Clinical Center of Kosova in Prishtina. Children with metabolic disease were excluded. Data on the laboratory work up and plain abdominal $\mathrm{x}$-ray were obtained from the patients history.

Results: 992 children with acute diarrhea were included. 17 were diagnosed with paralytic ileus. All ileuses were radiologically confirmed. All children has abdominal pain and distension. Only one had no vomiting $(1 / 17$ or $5.88 \%)$. The mean value for sodium was 136 and of potassium 3.59 with variations between 128 and 167 for sodium and 2.1 and 6.3 for potassium. In one child dehydration was hypernatremic. The mean value for blood urea was 3.30 and 71.33 for creatinine, with maximum values in the severely dehydrated children of 6.5 for urea and 270 for creatinine. $\mathrm{pH}$ in patients was between 7.25 and 7.44 with mean value of 7.34 .

Conclusion: Theonset of abdominal pain, distension and vomiting in children with acute diarrhea should prompt for exclusion of the paralytic ileus.

Key words: Gastroenteritis, acute, paralytic ileus

\section{3}

\section{PORTAL VENOUS GAS DETECTED BY ABDOMINAL ULTRASOUND IN THE DIAGNOSIS OF SUSPECTED NECROTIZING ENTEROCOLITIS}

\section{J. Mulvany}

\section{Neonatology, Rotunda Hospital, Dublin, Ireland}

Aim: To prospectively assess the validity of detecting portal venous gas on abdominal ultrasound (PVGUS) in the diagnosis of suspected NEC.

Methods: We conducted a prospective cohort study over a three-month period commencing in January 2010. We recruited 6 babies that presented with symptoms and signs suggestive of NEC as defined by the Walsh and Kliegman ${ }^{2}$ staging criteria. All babies had a plain abdominal x-ray performed at presentation. We followed standard protocols for the management of NEC. All babies had an abdominal ultrasound to detect portal venous gas within 48 hours of presentation using a Siemens Sonoline G60 ultrasound machine with a C-80 curvilinear 